\section{Correlated disorder in rainbow \\ metamaterials for vibration attenuation}

\author{
A.T. Fabro ${ }^{1}$, H. Meng ${ }^{2}$ and D. Chronopoulos ${ }^{2}$
}

\begin{abstract}
Metastructures are typically composed of periodic unit cells designed to present enhanced dynamic properties in which either single or multiple resonators are periodically distributed. Even though the periodic metamaterials can obtain bandgaps with outstanding vibration attenuation, the widths of bandgaps can still be narrow for some practical applications. Rainbow metamaterials have been proposed based on gradient or random profiles to provide further improved attenuation. Nonetheless, the effects of correlated random disorder on their attenuation performance remains an open challenge. This work presents an investigation on the effects of correlated disorder on the vibration attenuation of rainbow metamaterials. An analytical model using the transfer matrix approach is used to calculate the receptance functions in a finite length metastructure composed of evenly spaced non-symmetric resonators attached to a beam with $\Pi$-shaped cross-section, thus a multi-frequency metastructure. The correlated disorder is modelled using random fields and an analytical expression of the Karhunen-Loève expansion is used such that spatial correlation on the resonator properties is modified by various correlation lengths, i.e., the level of spatial smoothness. Individual samples of random fields are used to investigate the effects of the correlated disorder in the vibration attenuation of a multi-frequency metastructure. It is shown that the bandgap can be further widened when compared to uncorrelated disorder. The obtained results indicates that a combination of the gradient profile with some level of disorder, typically resulting from random fields with larger correlation lengths, tends to give improved vibration attenuation when compared to a optimized gradient rainbow metamaterial. It opens new and innovative ways for the design of broadband rainbow metastructures for vibration attenuation.
\end{abstract}

\title{
Keywords
}

Random field, Karhunen-Loève expansion, wave trapping, wave propagation

\section{Introduction}

Metastructures have been used for several engineering applications including vibration attenuation (1), in which the enhanced dynamics coming from periodicity and local resonators can be explored in several ways for improved broadband performance (2) . By using the concept of locally resonant materials, first introduced in acoustics by Liu et al. (3), significantly improved attenuation performance can be achieved when compared to the traditional single resonator design (4), while keeping the same mass ratio (5). Consequently, most of the proposed metastructures are periodic structures in which either single or multiple resonators configuration are periodically distributed. Despite of its improvement, the width of the bandgap can still be narrow for some practical applications.

Recently, the use of rainbow metastructure has been gaining attention and it is an interesting concept for achieving broad-band wave attenuation. Originally proposed in the context of optical waves (6), it relies on a spatial gradual transition of the local frequencies in such a way that the band gap gap can be further widen. This concept has been developed for acoustics (7), ultrasonics (8) and structural vibration $(9 ; 10)$. Moreover, Meng et al. (11) have shown that optimal spatial distribution of the tuning frequency of each resonator can be further explored for different performance criteria in multi-frequency metamaterials. Similarly, this concept has been extended to resonant piezoelectric based metastructures (12).

In order to enlarge the bandgap of metamaterials, a deviation from the typical periodic design, using near-periodic or quasi-periodic approaches is capable to significantly improving the dynamic performance of metastructures. Chen and Wang (13) have shown improved attenuation bands with localization of wave motion in elastic waves of along disordered and quasiperiodic, achieved using Fibonacci sequence, phononic crystals. Recently, Timorian et al. (14) have explored Fibonacci sequence based quasi-periodic in more details highlighting its attenuation performance. It has also been shown that this approach can be extended to $2 \mathrm{D}$

\footnotetext{
${ }^{1}$ Department of Mechanical Engineering, University of Brasilia, Brasília-DF, Brazil

${ }^{2}$ Institute for Aerospace Technology \& The Composites Group, University of Nottingham, Nottingham, UK
}

\section{Corresponding author:}

Adriano Todorovic Fabro, University of Brasília, Department of Mechanical Engineering - FT, Campus Darcy Ribeiro - Asa Norte, 70910-900, Brasília-DF, Brazil.

Email: fabro@unb.br 
quasi-periodic pattern using the Thue-Morse morphism sequence (15).

The investigation of the effects of randomness in wave propagation is not new. Scott (16) has studied the effects of scattering and energy flow in random media, assuming a wave equation with wavenumber plus a random term given by a stochastic process considering spatial correlation. Correlated disorder has also been investigated in long range spring-mass chain for DNA modelling (17) and extended acoustic waves (18). In addition, it has been known for decades this kind of near-periodicity can be designed for enhanced vibroacoustic attenuation performance $(19 ; 20 ; 21 ; 22)$. Similarly, randomness and break of periodicity can also be used to modify the performance of metastructures. For instance, Ruzzene and Baz (23) have shown extended the stop bands into adjacent propagation zones using disorder caused by the variance in the activation temperature of memory shape alloy inserts in rods. Achaoui et al. (24) have shown that for surface acoustic waves, Bragg scattering type of band gaps are less resilient to randomness than the locally resonant on two-dimensional pillars with hexagonal and honeycomb lattice symmetries. Sugino et al. (5) investigated the effects of randomness in both natural frequency and positioning of the resonators, suggesting that minimizing the variability, or the statistical dispersion of the randomness, is always beneficial for band gap widening. In contrast, Celli et al. (25) have shown random disorder can be used for band gap widening. This suggests that some underlying physics has not yet been fully explored. Notably, in these cases only the effect of uncorrelated disorder have been investigated, i.e., no spatial correlation or correlated disorder is taken into account in the properties of metastructure. Nonetheless, the effects of correlated random disorder, i.e., spatial correlation on the resonator properties, on the bandgap widening and maximum attenuation remains unexplored, to the best of the authors knowledge, and an open challenge to be addressed.

The aim of this work is to present an investigation of the effects of correlated disorder on the vibration attenuation performance in rainbow metamaterials. It is shown that the bandgap can be further widened when compared to uncorrelated disorder in the resonators tuning frequency. The obtained results indicates that a combination of the gradient profile with some level of disorder tends to give improved vibration attenuation even when compared to a optimized gradient rainbow metamaterial. This paper is organized as follows. The next section briefly introduces metastructure with nonsymmetric resonators for rainbow design, based on a previously proposed design $(11 ; 10)$. Then, the correlated disorder model based on an analytical solution of the Karhunen-Loève expansion is presented. Subsequently, numerical results are given and the influence of the correlation in the metastructure disorder is discussed based on the presented random field model in terms of single sample and in terms of a stochastic analysis. Finally, the last section gives some concluding remarks and further steps.

\section{Metamaterial beam with non-symmetric resonators}

In this section, the metastructure model is briefly presented. It is assumed a Euler-Bernoulli beam theory for both the baseline host structure and the cantilevermass resonator. Each resonator is modelled as a beam point attached to the baseline structure at one end and a lumped mass with negligible inertia at the other. They act independently that it is possible to create two separate band gap regions, consequently broadening the total attenuation band. This analytical model has been previously proposed and validated by experimental results $(10 ; 11 ; 26)$ and it is briefly presented here. Displacements of the П-shaped unit cell or segment are given as (10)

$$
\begin{aligned}
W_{n, l} & =\boldsymbol{\Lambda}_{n, l} \mathbf{a}_{n, l} \\
W_{n, r} & =\boldsymbol{\Lambda}_{n, r} \mathbf{a}_{n, r}
\end{aligned}
$$

where the subscripts $l$ and $l$ stands for the left and right ends of the $n$-th segment at $x_{n}$ and $x_{n r}=x_{n}+p_{n} L_{d}$, respectively, where $\boldsymbol{\Lambda}_{n, l}=$ $\operatorname{diag}\left\{\mathrm{e}^{-\mathrm{i} k\left(x-x_{n}\right)}, \mathrm{e}^{-k\left(x-x_{n}\right)}, \mathrm{e}^{\mathrm{i} k\left(x-x_{n}\right)}, \mathrm{e}^{k\left(x-x_{n}\right)}\right\}, \quad \boldsymbol{\Lambda}_{n, r}=$ $\operatorname{diag}\left\{\mathrm{e}^{-\mathrm{i} k\left(x-x_{n r}\right)}, \mathrm{e}^{-k\left(x-x_{n r}\right)}, \mathrm{e}^{\mathrm{i} k\left(x-x_{n r}\right)}, \mathrm{e}^{k\left(x-x_{n r}\right)}\right\}$,

$\operatorname{diag}\{\cdot\}$ stands for diagonal matrix, $\mathbf{a}_{n, l}=$ $\left[\alpha_{n, l}, \beta_{n, l}, \chi_{n, l}, \varepsilon_{n, l}\right]^{T}$, with $\alpha_{n, l}, \beta_{n, l}, \chi_{n, l}$ and $\varepsilon_{n, l}$ are the wave amplitudes at the left side $l$ of the $n$-th segment and $\mathbf{a}_{n, r}=\left[\alpha_{n, r}, \beta_{n, r}, \chi_{n, r}, \varepsilon_{n, r}\right]^{T}$ are the wave amplitudes at the right side $r$ of the $n$-th segment and $p_{s}$ is a factor for the positioning of the resonator along the unity cell. The geometrical parameters of the $n$-th segment are shown in Figure 1 and a schematic representation of the full metastructure is shown in Figure 2. Additionally, $k=\left(\rho A / E I_{z}\right)^{1 / 4} \sqrt{\omega}$, where $A$ is the cross section area, $I_{z}$ is the area moment of inertia of the $\Pi$-shaped beam, $\rho$ is the density of the rainbow metamaterial, $L_{d}$ is the length of the unity cell.

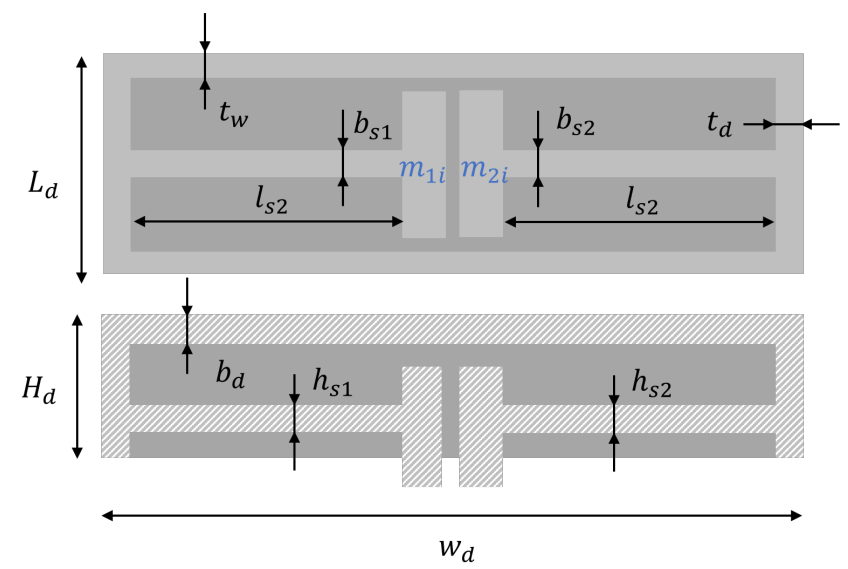

Figure 1. Schematic representation of a single unit cell of the metastructure with corresponding geometrical parameters. Tip masses parameters are shown in blue. 


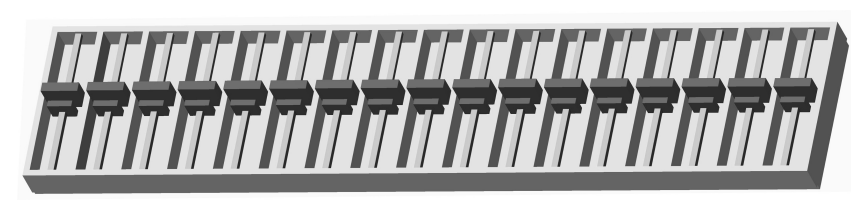

Figure 2. Schematic representation of full metastructure with non-symmetric resonators.

A transfer matrix approach can be used to relate the first to the $(n+1)$-th segment as (10)

$$
\mathbf{a}_{n+1, l}=\mathbf{\Xi}_{n} \mathbf{a}_{1, l},
$$

where $\boldsymbol{\Xi}_{n}=\mathbf{T}_{n} \mathbf{T}_{n-1} \ldots \mathbf{T}_{1}$. The transfer matrix for each individual unity cell depends on the dynamics of each unit cell whose detailed description is given in (10). This formulation can be used to calculate both free wave propagation and forced response. In this work, the transfer receptance function of the metastructure is given by

$$
\mathcal{H}(\omega)=20 \log _{10}\left|\frac{\left.W(\omega)_{q, r}\right|_{x=L}}{F}\right|,
$$

which is the displacement at the right side after $q$ due to a point unit force at the left side of the first segment. It can be similarly defined for the receptance along the whole metastructure length.

\section{Nearly-periodic metastructure}

For a periodic metastructure, the transfer matrix $\mathbf{T}_{n}$ is identical for every segment and a wavenumber $k_{n}$ can be defined from the propagation constant $\mu_{n}=\exp \left(-\mathrm{i} k_{n} L_{d}\right)$ such that $\left|\mathbf{T}_{n}-\mu_{n} \mathbf{I}\right|=0$, typically applied in 1D periodic systems (1) . For nearly periodic structures, an equivalent wavenumber $k_{T}$ can be defined from the propagation constant $\mu_{T}=\exp \left(-\mathrm{i} k_{T} L\right)$ of the finite length metamaterial with spatially varying properties as $(10 ; 27)$

$$
\left|\boldsymbol{\Xi}-\mu_{T} \mathbf{I}\right|=0,
$$

This definition can be also interpreted as the wavenumber in the case that the finite rainbow metamaterial is a periodic unit of an infinite complex beam. In this sense, it represents the total phase change and attenuation of a travelling wave over $q$ unit cells and this interpretation can be consequently used as a tool to understand the wave-like behaviour of nonperiodic structures. It is important to highlight that the total phase and attenuation change $k_{T} L$, which has real and imaginary parts, is not generally equal to the contribution of the phase and attenuation change at each segment in the near periodic metastructure, i.e. $k_{T} L \neq \sum_{n=1}^{m} k_{n} L_{d}$. This is a consequence of the additional scattering created due to the presence of critical sections, also known as turning points (28), arising not only from from rapidly changing properties but also due to waves cutting-on/off or due to waves veering or locking (29). This effect is particularly relevant in metastructures and has been experimentally investigated from 3D printed metastructures and it is related to wave trapping at band gap frequency edges (30). In the case of slowly varying properties, this internal scattering is negligible, consequently $k_{T} L \approx$ $\sum_{n=1}^{m} k_{n} L_{d}$ can be assumed $(31 ; 32)$.

\section{Random field model of correlated disorder}

Spatially correlated randomness in nearly-periodic metastructures can be modelled using random fields (33), which are a multidimensional random processes using a probability measure. This kind of representation has the advantage of capturing the essential features of a complex random phenomenon in terms of a small number of physically meaningful and experimentally accessible parameters and has been recently used to represent spatially correlated variability of mechanical or geometrical properties of 3D structures $(34 ; 35 ; 27)$.

The Karhunen-Loève expansion (KLE) is used to describe second order random fields in terms of deterministic eigenfunctions and eigenvalues and correspoding uncorrelated random variables. This representation has been widely used for stochastic modelling structural dynamics for several decades (see e.g. the review by Schueller and Pradlwarter (36)). In the case of the one-dimensional exponentially decaying autocorrelation function

$$
C(\tau)=\mathrm{e}^{-|\tau| / c_{l}},
$$

where $c_{l}$ is the correlation length and $\tau$ is the distance between two any points, the KLE has an analytical expression given by (37)

$$
H(x)=\sum_{i=1}^{\infty}\left[\xi_{1 i} \sqrt{\lambda_{i}} \frac{\sin \left(\mathrm{w}_{1 i} x\right)}{\alpha_{i}}+\xi_{2 i} \sqrt{\lambda_{i}} \frac{\cos \left(\mathrm{w}_{2 i} x\right)}{\beta_{i}}\right],
$$

valid in the interval $-L_{t} / 2 \leq x \leq L_{t} / 2$, where $L_{t}$ is the length of the domain considered, $\xi_{1 i}$ and $\xi_{2 i}$ are zeromean, unit standard-deviation and independent random variables. In this work, $\xi_{i}$ is assumed to be a Gaussian random variable for sake simplicity, but non-Gaussian random fields can be generated following the same analytical structure without distorting the targeted correlation function using iterative scheme $(38 ; 39 ; 40)$. The eigenfunctions are normalized by

$$
\alpha_{i}=\sqrt{\frac{L_{t}}{2}-\frac{\sin \left(\mathrm{w}_{1 i} L_{t}\right)}{2 \mathrm{w}_{1 i}}}, \beta_{i}=\sqrt{\frac{L_{t}}{2}+\frac{\sin \left(\mathrm{w}_{2 i} L_{t}\right)}{2 \mathrm{w}_{2 i}}},
$$

and the eigenvalues are given by $\lambda_{1 j}=2 \mathrm{c} / \mathrm{w}_{1 i}^{2}+\mathrm{c}^{2}$, $\lambda_{2 j}=2 \mathrm{c} / \mathrm{w}_{2 i}^{2}+\mathrm{c}^{2}, \mathrm{c}=1 / c_{l}$ and $\mathrm{w}_{1 j}$ and $\mathrm{w}_{2 j}$ are the $j^{\text {th }}$ roots of the transcendental equations $\mathrm{c} \tan \mathrm{w}_{1 j}+\mathrm{w}_{1 j}=0$ and $\mathrm{w}_{2 j} \tan \mathrm{w}_{2 j}-\mathrm{c}=0$, respectively.

The series expansion can be ordered in descending order of magnitude of the eigenvalues and thus truncated to a finite number of terms $N_{K L}$. As a rule of thumb, $N_{K L}$ can be chosen such that $\lambda_{N_{K L}} / \lambda_{1}<$ 0.1 depending on the correlation length $c_{l}$, such that Eq. 6 can be rewritten as $H(x)=\sum_{i=1}^{N_{K L}} \xi_{i} \sqrt{\lambda_{i}} f_{i}\left(\mathrm{w}_{i} x\right)$. For longer correlation lengths, the eigenvalues decrease 
more rapidly thus fewer terms are needed to accurately represent the series.

The KLE can also be interpreted from a spectral point of view. In this sense, the coefficients $\mathrm{w}_{i}$ are the spatial frequency related to each term of the KLE series. Thus, a wavelength for each corresponding eigenfunction $f_{j}\left(\mathrm{w}_{j} x\right)$ can be defined as (41)

$$
l_{i}^{K L}=\frac{2 \pi}{\mathrm{w}_{i}} .
$$

Therefore, it is possible to compare the scale of the spatial fluctuation $l_{i}^{K L}$ to the size of the metastructure unit-cell $L_{d}$. Note that this depends directly on the correlation length $c_{l}$. For each term of the series, the $i$-th eigenvalue $\sqrt{\lambda_{i}}$ gives the weight of the scale of the spatial disorder introduced by its corresponding eigenfunction and dominated by $l_{i}^{K L}$.

Furthermore, it is possible to note from Eq. 6 that for each individual random field sample this weighting is random and given according to the probability density function of $\xi_{i}$. Thus, different random field samples with the same statistical features can introduce different levels of scales of spatial fluctuation, and consequently different levels of spatial disorder.

Finally, it should be noted that although an analytical formulation of the KLE expansion is used in this work, different correlation functions can also be directly applied by solving the KLE numerically, even though the analytical formulation gives significantly better convergence when compared to a numerical solution (41). (42) have shown that the correlation length plays a much bigger role than the shape of the correlation function in the statistics of the response, thus only the effects of the correlation length is investigated in this paper.

\section{Correlated disorder in the mistuned resonators}

The correlated disorder is introduced by varying the tip masses of the resonators according to

$$
\mathbf{m}_{1,2}=m_{01,2}\left(\mathbf{1}+\sigma_{m 1,2} \tilde{\mathbf{h}}_{1,2}\right),
$$

\footnotetext{
where $m_{01,2}$ is the nominal values of the resonators tip masses in the periodic design, $\mathbf{1}$ is a vector $q \times 1$ vector filled with 1 and $\sigma_{m}$ controls the level of statistical dispersion of the random vectors $\tilde{\mathbf{h}}_{1,2}$. The latter is obtained by discretizing the Gaussian homogeneous random field $H(x)$, Equation 6, at the attachment position of the resonators on each unit-cell of the finite length metastructure, which is assumed to be at its middle point. When applied to a classical finite element discretization, this is known as the mid-point method of random field discretization (43) and typically it used such that the distance between two points to be four times smaller than the random field correlation length, as a rule of thumb (44).
}

A mass ratio is defined as the ratio of the total tip mass of each resonator $m_{1 i}$ and $m_{2 i}$ over the total mass of the metastructure $M_{t}$, i.e. (11)

$$
\mathcal{R}=\sum_{i=1}^{q}\left(m_{1 i}+m_{2 i}\right) / M_{t},
$$

where $M_{t}$ is the total mass of the metastructure. Increasing the mass ratio $\mathcal{R}$ typically widens the band gap in periodic metastructures (5), however it has the drawback of increasing the total mass of the structure. At the $i$-th segment, the tip masses of each resonator are given by $m_{1 i}$ and $m_{2 i}$. A reference mass is defined as the unit mass of the $\Pi$-shaped beam with parallel insertions as $m_{r}=M_{t} / q$.

Moreover, Stefanou and Papadrakakis (45) have shown that the individual samples generated from the KLE are in general not ergodic in mean nor in spatial correlation, i.e. the ensemble statistics might differ from the spatial domain statistics. Consequently, each individual sample might either not have zero spatial average equals over the metastructure, which will modify the added mass due to the resonators, nor keep the same correlation length. However, this effect will not affect the resulting analysis of this paper.

Finally, the uncorrelated disorder corresponds to the case in which the correlation length equal zero, i.e., the values of the tip masses are statistically independent, giving a white-noise type of spatial profile. For increasing correlation length, the spatial variation of masses becomes increasingly smooth, such that for infinitely large correlation correspond to a homogeneous profile, thus the periodic design.

\section{Numerical results}

In this section, the numerical results and analysis are presented in two subsections. The first concerns the effects of correlated disorder produced from a single sample of a random field. Variation in the resonators frequencies in created by spatially changing their tip masses. The second presents the effects of the correlation in the statistics of the transfer receptance.

For all of the examples, it is assumed Young's modulus $E=1.8 \mathrm{GPa}$, mass density $\rho=930 \mathrm{~kg} / \mathrm{m}^{3}$ and loss factor $\eta=0.03$. The geometrical parameters, presented in Figure 1 for a single segment of the metastructure, are the height $H_{d}=10 \mathrm{~mm}$, width $w_{d}=51 \mathrm{~mm}$, side wall thickness $t_{d}=2 \mathrm{~mm}$, backplate thickness $b_{d}=5 \mathrm{~mm}$, plate insertion thickness $t_{w}=2 \mathrm{~mm}$, distance between plate insertions $L_{d}=15 \mathrm{~mm}$ and a total of $q=17$ segments, or unit-cells, such that the metastructure has a total length $L_{t}=0.255 \mathrm{~mm}$. Moreover, each cantilever beam has height $h_{s 1}=1.4 \mathrm{~mm}$ and $h_{s 2}=2.3 \mathrm{~mm}$, width $b_{s 1}=1.9 \mathrm{~mm}$ and $b_{s 2}=2.3 \mathrm{~mm}$ and length $l_{s 1}=l_{s 2}=$ $21.2 \mathrm{~mm}$. The frequency range under consideration is from $100 \mathrm{~Hz}$ to $500 \mathrm{~Hz}$ with steps of $0.5 \mathrm{~Hz}$. 


\section{Rainbow correlated disorder: sample spatial profile}

In this section, a single sample of the presented random field is used for different correlation lengths $c_{l}=4 L_{t}$, $c_{l}=L_{t}, c_{l}=0.25 L_{t}$ and $c_{l} \rightarrow 0$. The latter represents the uncorrelated case as the random field tends to a white noise type of stochastic process. In practice, it is generated by performing the KLE with a very small correlation length $\left(c_{l}=0.001 L_{t}\right)$. Results are compared to the periodic case, i.e., identical masses at each segment along the metastructure, and to a optimized gradient profile for a rainbow metamaterial.

For the correlated cases, the mass profile is assumed to follow $\mathbf{m}_{1}=m_{01}\left(\mathbf{1}+\sigma_{m 1} \tilde{\mathbf{h}}_{1}\right)$ and $\mathbf{m}_{2}=m_{02}(\mathbf{1}+$ $\left.\sigma_{m 2} \tilde{\mathbf{h}}_{2}\right)$, where $m_{01}=0.053 m_{r}, m_{02}=0.032 m_{r}, \sigma_{m 2}=$ $0.01 / 0.053 \approx 0.19$ and $\sigma_{m 1}=2 \sigma_{m 2}$. These values are assumed such the average mass value is approximately close to the reference optimized case, given by $m_{1 i}=$ $A_{1}+B_{1} \sin \left(C_{1} x+D_{1}\right) ;$ with $A_{1}=0.056727 m_{r}, B_{1}=$ $0.024099 m_{r}, \quad C_{1}=-1.511657, \quad D_{1}=0.047384, \quad$ and $m_{2 i}=A_{2}+B_{2} \sin \left(C_{2} x+D_{2}\right)$, with $A_{2}=0.053924 m_{r}$ $B_{2}=0.024125 m_{r}, C_{2}=-0.93883$ and $D_{2}=-0.97644$. More details on the optimization approach are found in (11).

The tip mass profiles are shown in Figure 3 for each considered case, all of them are obtained using $N_{K L}=6$ KLE terms but $c_{l} \rightarrow 0$, in which $N_{K L}=100$. Identical random field samples are used for both tip masses in each segment, i.e. $\tilde{\mathbf{h}}_{1}=\tilde{\mathbf{h}}_{2}$.
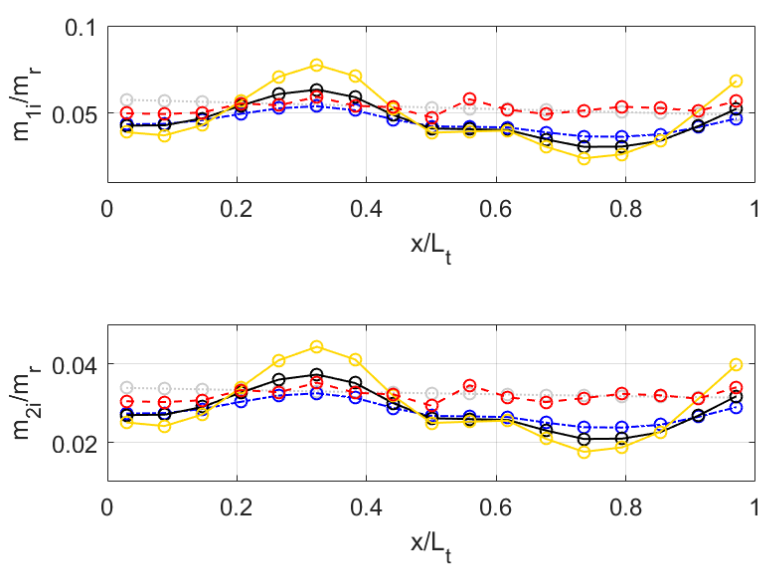

Figure 3. Resonator mass profile $m_{1 i}$ (upper) and $m_{2} i$ (lower) along the metastructure considering the periodic design (full gray line), optimal rainbow deterministic profile (dotted gray line) and rainbow correlated disorder with $c_{l}=4 L_{t}$ (dash-dotted blue line) $c_{l}=L_{t}$ (full black line), $c_{l}=0.25 L_{t}$ (full yellow line) and $c_{l} \rightarrow 0$ (dashed red line).

Note that longer correlation lengths generate smoother spatial profiles, i.e. low spatial frequency components are dominant for the cases with long correlation length. This is similar to a gradient profile with slight perturbation along the metastructure length. In the case with $c_{l} \rightarrow 0$, only these perturbations are dominant so the spatial profile is rougher and the overall amplitude of the spatial profile is smaller.
Table 1 summarizes the corresponding mass ratio $\mathcal{R}$, defined by Equation 10, for each sample of disorder, periodic and optimized case. The mass ratios for the correlated rainbow metastructure are only slightly higher than the periodic, gradient optimized and uncorrelated cases, as a consequence of the nonergodicity of the individual KLE samples.

Table 1. Rainbow metastructure mass ratio $\mathcal{R}$ for each sample of disorder, periodic and gradient optimized case.

\begin{tabular}{c|c}
$c_{l} / L_{t}$ & $\mathcal{R} \%$ \\
\hline 4.00 & 10.1 \\
1.00 & 10.3 \\
0.25 & 9.3 \\
uncorrelated & 8.4 \\
periodic & 8.5 \\
optimized & 8.6
\end{tabular}

The same sequence of sampled Gaussian random variables $\xi_{i}$ is used for every case such that the effects of the correlation length $c_{l}$ can be more clearly analyzed. Figure 4 presents the KLE normalized eigenvalues and normalized random samples as a function of the corresponding eigenfunctions wavelength $l_{i}^{K L}$ normalized by the unit cell length $L_{d}$ for correlation length $c_{l}$.

For all of the cases, the random variable $\xi_{i}$ introduces a random fluctuation to each deterministic term of the series. For larger the correlation length, larger wavelengths $l_{i}^{K L}$ have a higher chance to preponderate over the random field sample. This has the effect of given a smoother variation for larger correlations lengths and, in turn, a rougher profile for shorter correlation lengths. In the smoother case $c_{l}=4 L_{t}$, Figure $4(\mathrm{a})$, the most important KLE term has a wavelength which is more than 100 times larger than the unit cell, i.e. $l_{1}^{K L} \approx$ $100 L_{d}$, which is similar to the optimal gradient profile obtained by Meng et al. (11). In this case $N_{K L}=6$ terms is enough for the KLE series convergence, following the rule of thumb $\lambda_{N_{K L}} / \lambda_{1}<0.1$, and also to avoid spatial aliasing, which might appear when the random field wavelength $l_{i}^{K L}<2 L_{d}$. However, this truncation decreases the overall variance of the random field (46). Similar effects are obtained for $c_{l}=L_{t}$ and $c_{l}=$ $0.25 L_{t}$, in which KLE terms with smaller wavelength get increasingly importance in the series. Recall that the uncorrelated case is represented by making $c_{l} \rightarrow 0$. Nevertheless, such interpretation from KLE series can still be used, and it is presented in Figure 4(d). However, unlike the correlated cases, all of the terms in the KLE have equal weight thus giving a rougher spatial profile with high spatial frequency content. In this case, $N_{K L}=100$ terms are used in the series which does not follow the rule of thumb $\lambda_{N_{K L}} / \lambda_{1}<0.1$ but it is the maximum number of terms allowed such that no spatial aliasing is introduced. This is equivalent to a band limited white noise in stochastic processes and it is inherent in metastructures with unit cells. 


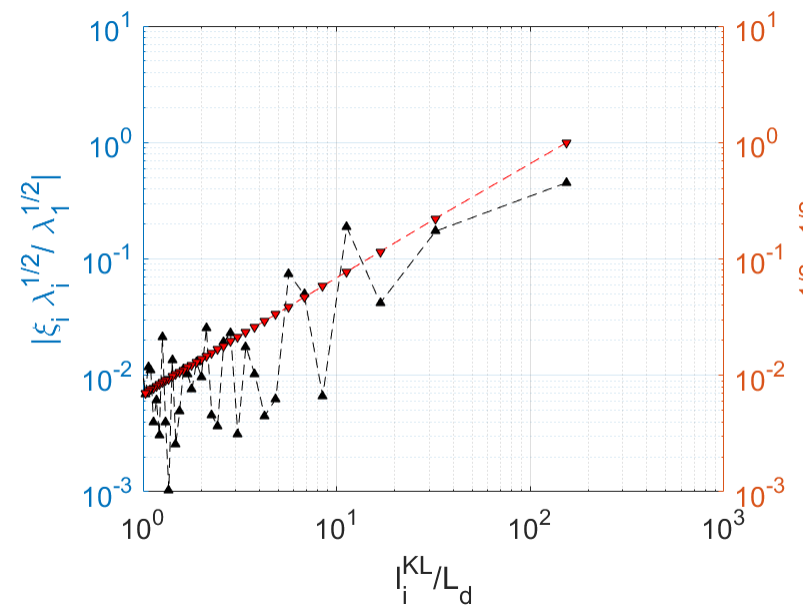

(a) $c_{l}=4 L_{t}$

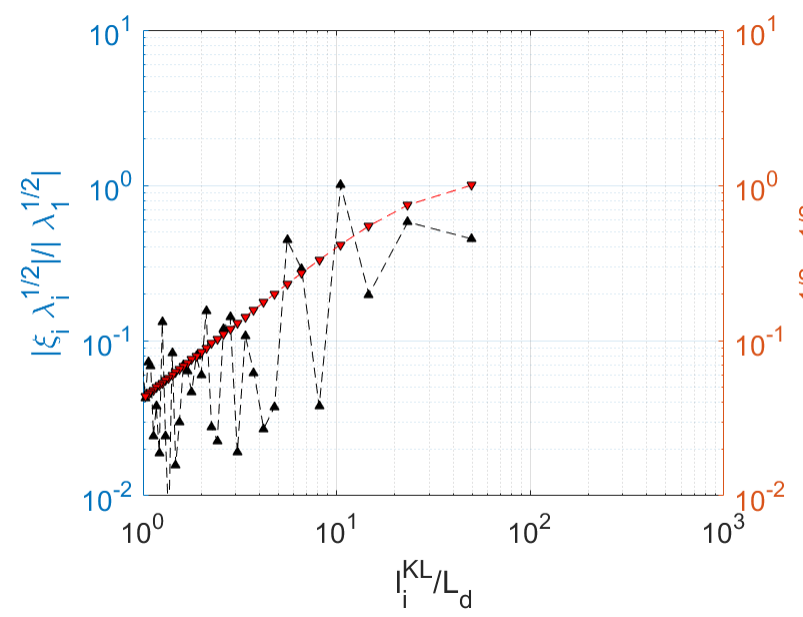

(c) $c_{l}=0.25 L_{t}$

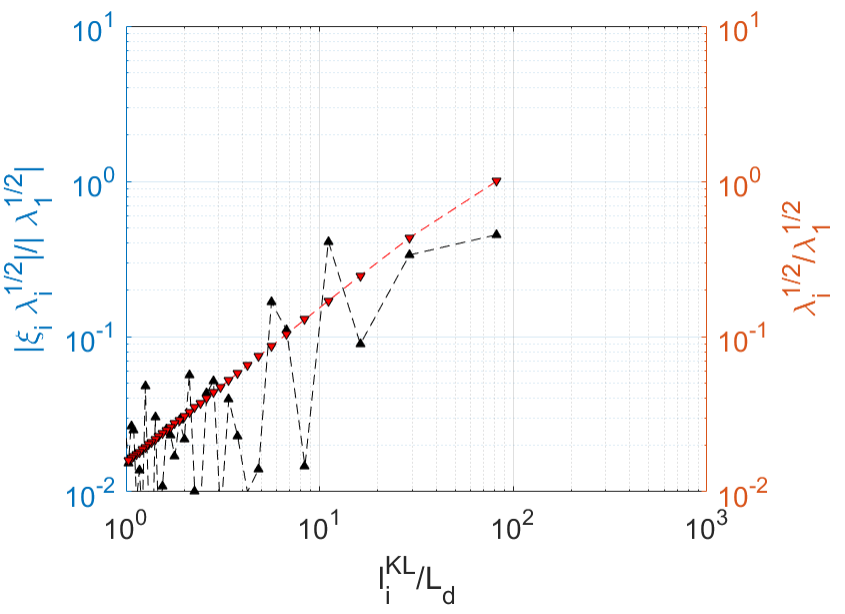

(b) $c_{l}=L_{t}$

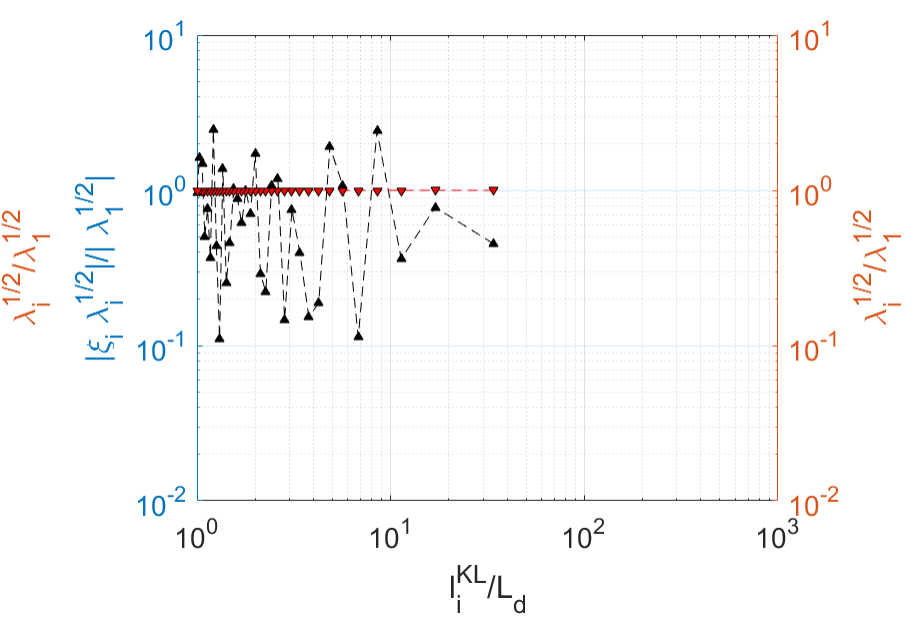

(d) $c_{l} \rightarrow 0$

Figure 4. Karhunen-Loève normalized eigenvalues (right axis) and normalized random samples (left axis) as a function of the corresponding eigenfunctions wavelength $l_{i}^{K L}$ normalized by the unit cell length $L_{d}$ for correlation length $c_{l}$.

\section{Vibration attenuation}

In this section, the effects of the different types of disorder in the vibration response and in the equivalent wavenumber $k_{T}$ of the metastructure is investigated.

Figure 5 presents the amplitude of the transfer receptance of the metastructure considering the periodic design, a rainbow metastructure with gradient profile and a rainbow metastructure with correlated disorder assuming $c_{l}=4 L_{t}$. Two band gaps are created in the periodic design at the natural frequency of each resonator in the non-symmetric unit-cell design, 149 $\mathrm{Hz}$ and $423 \mathrm{~Hz}$. The optimal gradient rainbow design is the obtained by a optimization approach proposed by Meng et al. (11), using Genetic Algorithm and a maximum value based objective function. It is assumed two prescribed multi-frequency ranges as $140 \sim 160$ $\mathrm{Hz}$ and $410 \sim 430 \mathrm{~Hz}$ for the optimization strategy, which leads to wider band gaps but weaker vibration attenuation when compared to the periodic design, as seen in Figure 5. Similarly, the band gaps created by the rainbow correlated disorder design show a wider frequency range but a even weaker vibration attenuation. However, they also substantially reduce the amplitude of the vibration modes just after both band gap.

The effect of decreasing the correlation length $c_{l}$ in the amplitude of the transfer receptance are shown in Figure 6. The same case assuming $c_{l}=4 L_{t}$ is repeated for sake of comparison with shorter correlation lengths $c_{l}=L_{t}, c_{l}=0.25 L_{t}$ and $c_{l} \rightarrow 0$. Recall that the latter is considered here as a limiting case to the uncorrelated disorder and that these tip mass profiles are obtained keeping the same random samples $\xi_{i}$ but changing only the corresponding $\lambda_{i}$ eigenvalues and the KLE wavelength $l_{i}^{K L}$ of each eigenfunction $f_{i}\left(\mathrm{w}_{\mathrm{i}} x\right)$, as described in the previous section. From Figure 6, it is possible to note that by decreasing the correlation length, the attenuation band still presents a jagged profile in the receptance amplitude. This is not the case for $c_{l} \rightarrow 0$ because of the reduced variance in the tip masses profile due to the truncation in the KLE series (37). Moreover, Sugino et al. (5) have shown that increasing the variance level in uncorrelated disorder 


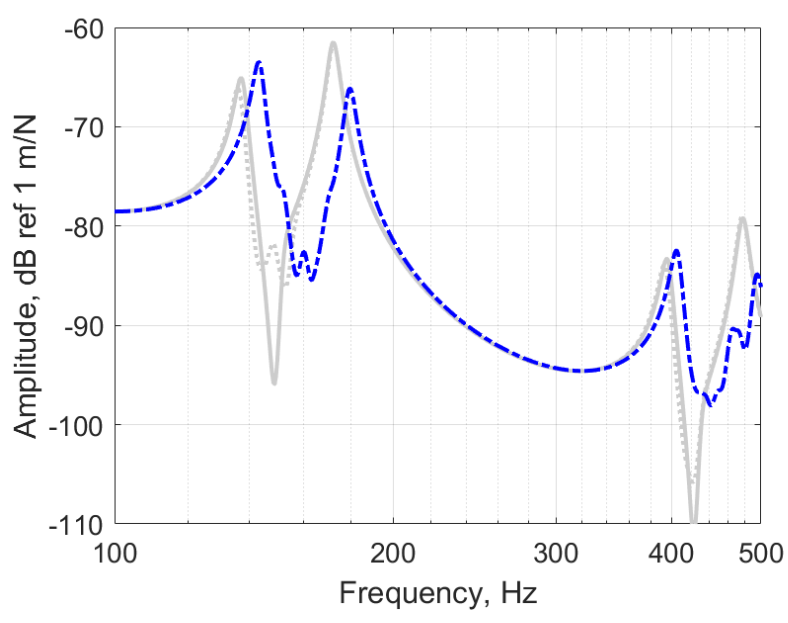

Figure 5. Amplitude of the transfer receptance of the metastructure considering the periodic design (full gray line), optimal rainbow gradient profile (dotted gray line) and rainbow correlated disorder with $c_{l}=4 L_{t}$ (full black line).

in one dimensional metastructures has the effect of

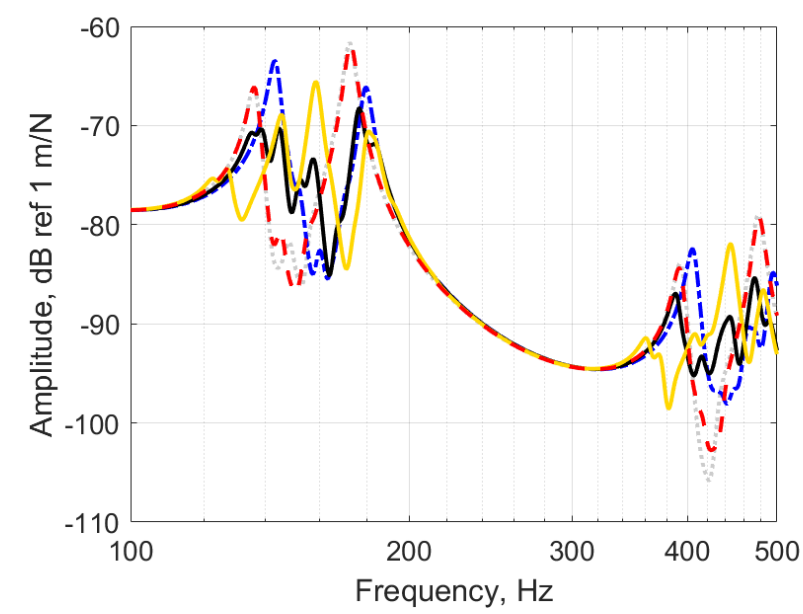

Figure 6. Amplitude of the transfer receptance of the metastructure considering the rainbow correlated disorder with $c_{l}=4 L_{t}$ (dash-dotted blue line) $c_{l}=L_{t}$ (full black line), $c_{l}=0.25 L_{t}$ (full yellow line) and $c_{l} \rightarrow 0$ (dashed red line).

This effect is also seen in Figure 7, which presents amplitude of the vibration modes at the edges of the band gaps is also reduced by up to $12 \mathrm{~dB}$.
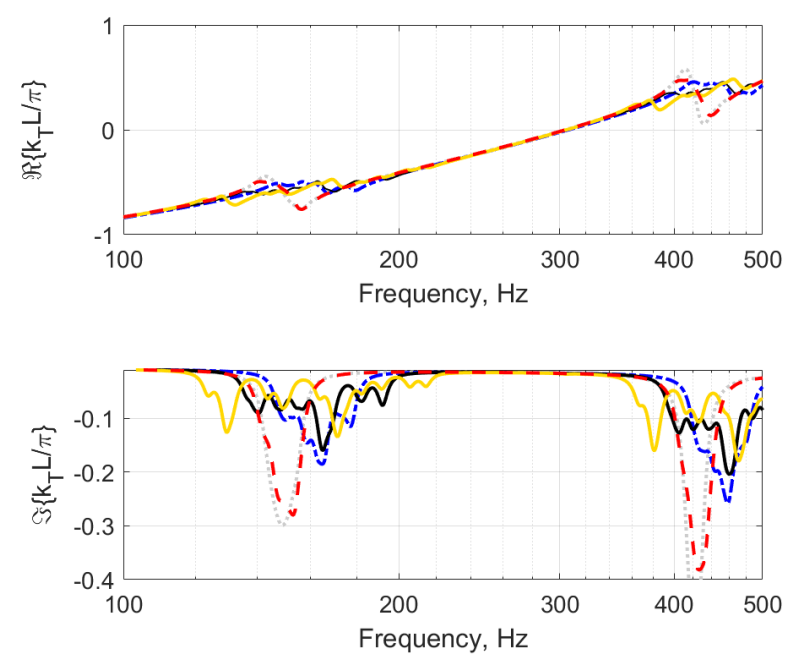

Figure 7. Real (upper) and Imaginary part (lower) of the equivalent wavenumber $k_{T}$ optimal gradient rainbow (dotted gray line) and rainbow correlated disorder with $c_{l}=4 L_{t}$ (dash-dotted blue line) $c_{l}=L_{t}$ (full black line), $c_{l}=0.25 L_{t}$ (full yellow line) and $c_{l} \rightarrow 0$ (dashed red line).

Beli et al. (30) have experimentally shown that this effect is attributed to wave trapping generated by turning points, which are created at the edges of the band gaps, leading to mode localization, as described by Luongo (28). This effect is confirmed by the operational deflection shapes (ODS) of the metastructure shown in Figure 8. Notice that in the periodic case, the band regions are well defined at the band gap edges as soon as the near-field due to the point excitation at $x=0$ is negligible, which is around $x / L_{t}=0.3$ for the lowest attenuation band and $x / L_{t}=0.1$ for the higher attenuation band. The wave trapping is clearly visible at the ODS in the optimal gradient rainbow metastructure, which has the effect of widening the attenuation band at $x / L_{t}=1$. A similar effect is seen for rainbow metamaterial with correlated disorder and correlation length $c_{l}=4 L_{t}$. In this case, the correlated disorder creates a similar gradient structure due to the large wavelengths $l_{t}^{K L}$ in the KLE series. For shorter correlation lengths, the shorter $l_{t}^{K L}$ widens further the band gap but significantly decreases the attenuation at $x / L_{t}=1$ due to the wave trapping in larger bands along the metastructure. Consequently, the vibration modes, which are well defined at the band gap edges for the periodic and optimal gradient rainbow metastructures, are significantly reduced in amplitude.

\section{Stochastic analysis}

In the previous section, the correlated disorder effects of a single sample of the proposed random field model is explored for different correlation lengths. Although similar physical effects are expected, different random samples can generate significantly different attenuation performances. This is highlighted in Figure 9, in which 


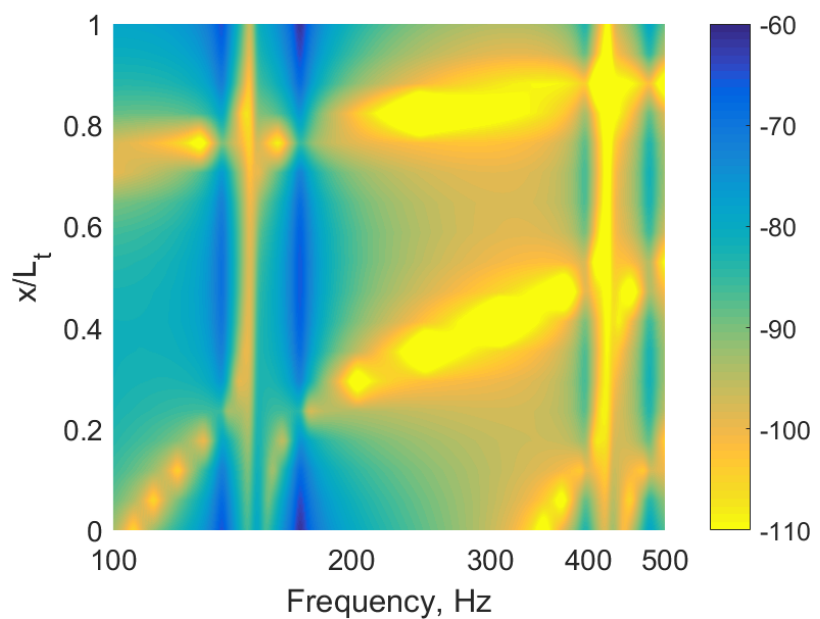

(a) periodic

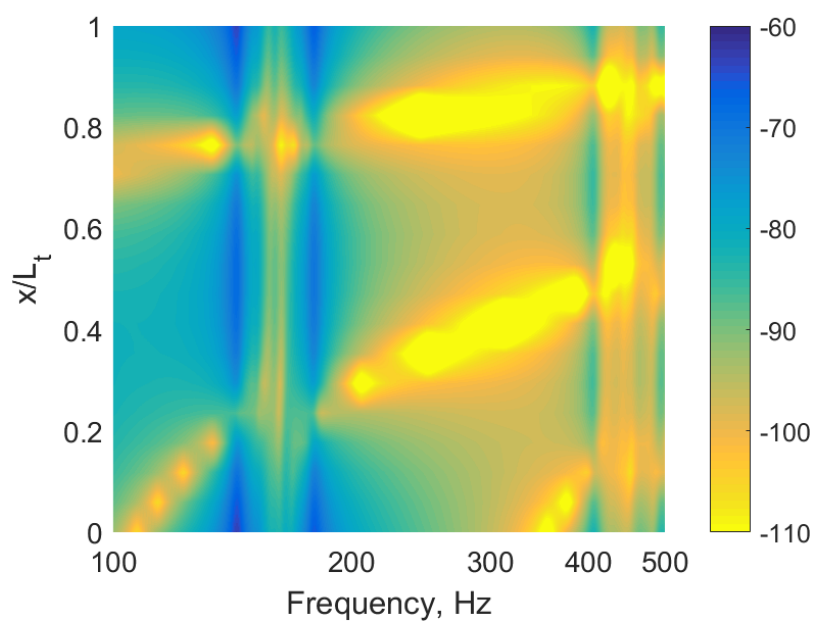

(c) $c_{l}=4 L_{t}$

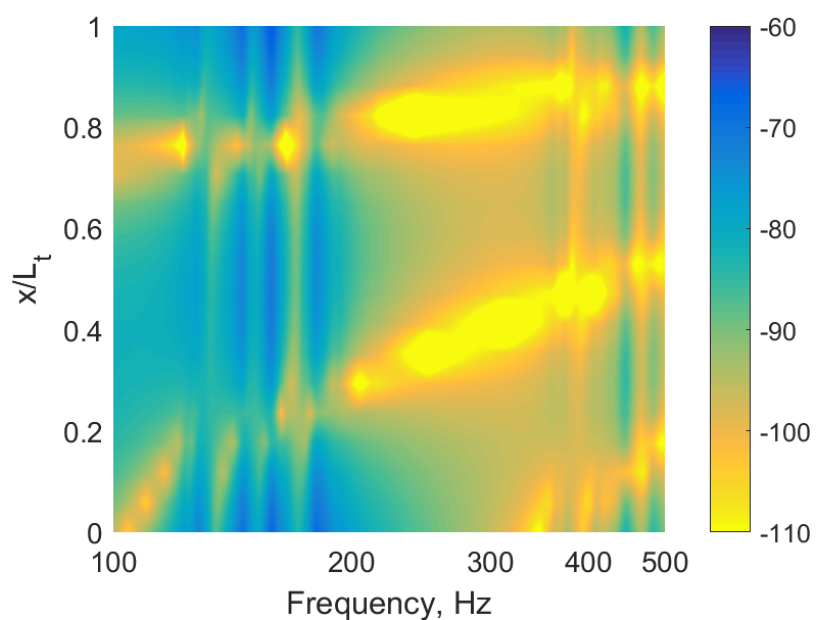

(e) $c_{l}=0.25 L_{t}$

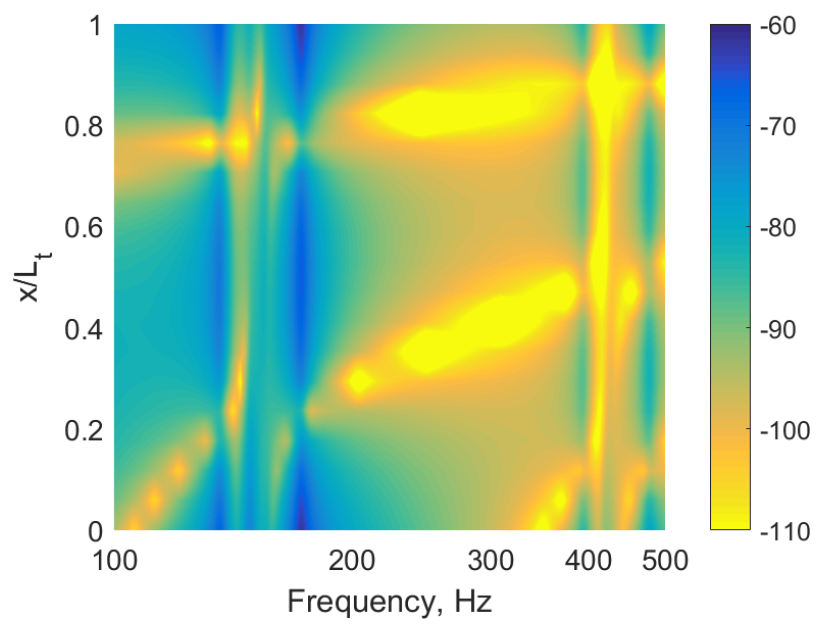

(b) optimal gradient rainbow

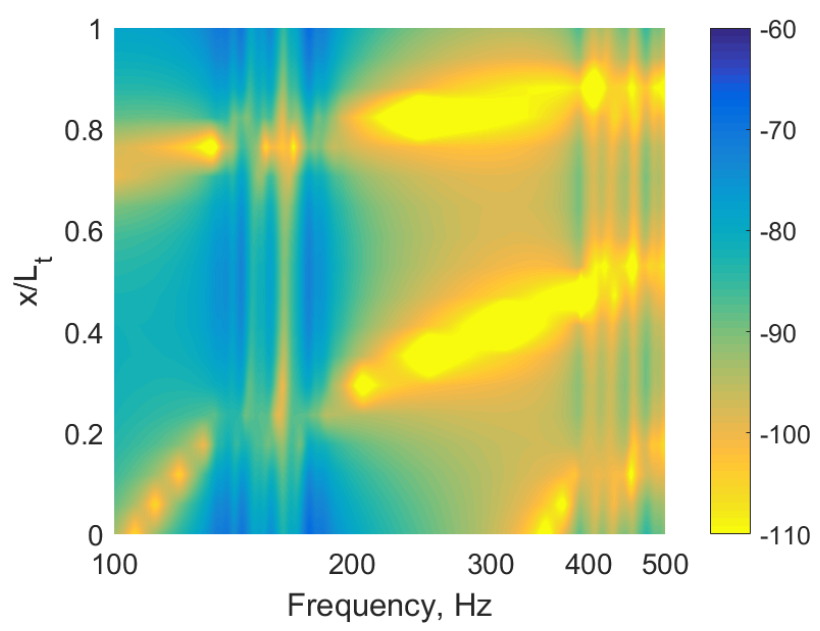

(d) $c_{l}=L_{t}$

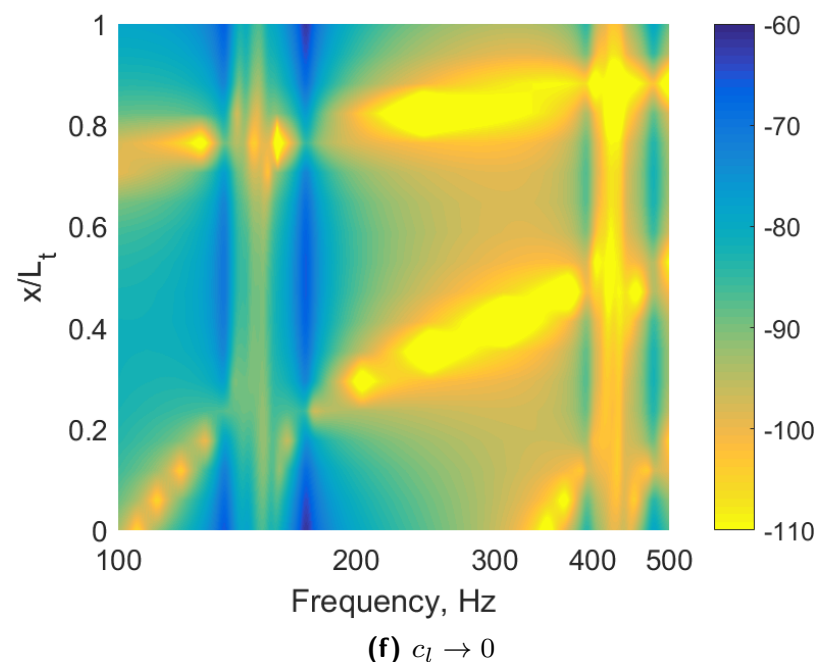

Figure 8. Amplitude of the receptance in $\mathrm{dB}$ re $1 \mathrm{~m} / \mathrm{N}$ along the metastructure considering the (a) periodic design, (b) optimal gradient rainbow and rainbow correlated disorder with (c) $c_{l}=4 L_{t}$, (d) $c_{l}=L_{t}$, (e) $c_{l}=0.25 L_{t}$ and (f) $c_{l} \rightarrow 0$. Point harmonic excitation at $x=0$.

four extra samples of random masses profile which are statistically equivalent, i.e. generated from the sample random field model, lead to different vibration responses for $c_{l}=4 L_{t}$. This effect is expected and has been ${ }_{512}$ experimentally observed by Beli et al. (30). 


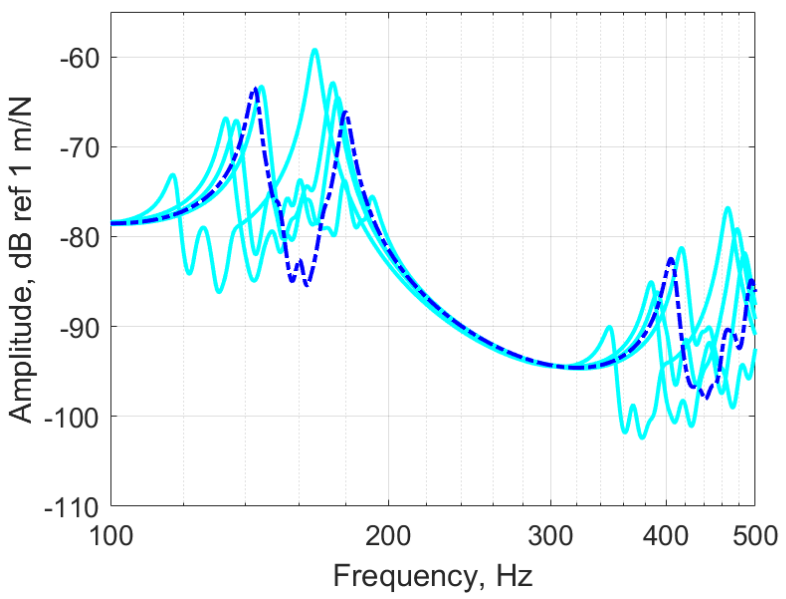

Figure 9. Amplitude of the transfer receptance of the metastructure considering the reference case (dash-dotted blue line) and four other independent random samples (cyan) of rainbow correlated disorder with $c_{l}=4 L_{t}$.

Although a virtually infinite number of random samples can be generated, limited by the used sampling algorithm (47), it is physically reasonable to expect that the response bounds are limited. To investigate these limits, Monte Carlo sampling is performed as the stochastic solver to calculate the mean value and the $5^{\text {th }}$ and $95^{\text {th }}$ percentile of the transfer receptance. Figures 10 and 11 show the results obtained for the amplitude of the transfer receptance the imaginary part of the equivalent wavenumber $k_{T}$ with 1,000 samples, which is enough for achieving mean value convergence (46). Notice that in both results, the percentile bounds for $c_{l} \rightarrow 0$ is much smaller when compared to the correlated cases due to the reduced variance in the random field caused by the truncation of the KLE series, as previously discussed.

Moreover, the mean value averages out the attenuation at the band gaps and clearly cannot be used a representative response of system. In addition, because only the tip masses are assumed as random variables, it is expected that the uncertainty in the response in only at the band gaps. This effect is clearly seen at the percentile bounds shown in Figure 11. It shows that, on average, the min value of $\Im\left\{k_{T}\right\}$, which given the maximum vibration attenuation, is very similar to the optimized gradient metamaterial, Figure 7, but significantly wider in frequency. It shows that the the correlated disorder can potentially achieve the same levels of vibration attenuation with wider frequency range.

The cases $c_{l}=4 L_{t}$ and $c_{l}=L_{t}$ show very similar percentile bounds, showing that significant attenuation can be achieved on average, while in the shorter correlation $c_{l}=0.25 L_{t}$ this is decreased significantly. This indicates that a combination of the gradient profile, which appears at the larger correlation lengths, with some level of disorder, tends to give improved vibration attenuation when compared with shorter correlation lengths.

\section{Concluding remarks}

This work presents an investigation of the effects of correlated disorder on the vibration attenuation performance in multi-frequency rainbow metamaterials. An analytical model using the transfer matrix approach is used to calculate the receptance in a finite length metastructure composed of evenly spaced nonsymmetric resonators attached to a beam with $\Pi$-shaped cross-section, thus a multi-frequency metastructure. The disorder is generated by imposing a spatial correlation,i.e., the level of spatial smoothness, on the random values of the tip masses of non-symmetrical resonators along the metastructure.

The analysis of individual samples of a random field model shows that it can potentially improve to overall vibration attenuation when compared to an optimized gradient rainbow metamaterial. It indicates that a combination of the gradient profile, which appears at the larger correlation lengths, with some level of disorder, tends to give improved vibration attenuation when compared with shorter correlation lengths.

The presented results show that the correlated disorder can be potentially used in optimization schemes for increased attenuation performance, by further widening band gap and also reducing the effects of the vibration modes at the band gap edges. It opens new and innovative ways for the design of broadband rainbow metastructures for vibration attenuation from additive manufacturing. It can also be potentially used in conjunction with smart structures applications, using piezoelectric shunt-based local resonators or shape memory alloys.

\section{Supplementary data}

We believe that the paper provides sufficient information for the results to be fully reproducible. In any case, any underlying research material related to this work can be directly requested to the corresponding author. Numerical simulation data for the metastructure in this paper in freely available at (26).

\section{Acknowledgements}

The authors would like to acknowledge the support acquired by the H2020 DiaMoND project (Grant Agreement ID:785859), the Brazilian National Council of Research CNPq (Grant Agreement ID: 420304/2018-5) and the São Paulo Research Foundation FAPESP ENVIBRO Thematic Grant (Grant Agreement ID: 2018/15894-0)

\section{References}

[1] Hussein MI, Leamy MJ and Ruzzene M. Dynamics of Phononic Materials and Structures: Historical Origins, Recent Progress, and Future Outlook. Applied Mechanics Reviews 2014; 66(4): 40802. DOI:10. 1115/1.4026911. URL http://dx.doi.org/10.1115/1. 4026911.

[2] De Rosa S, Desmet W, Ichchou M et al. Vibroacoustics of periodic media: Multi-scale modelling and design of 


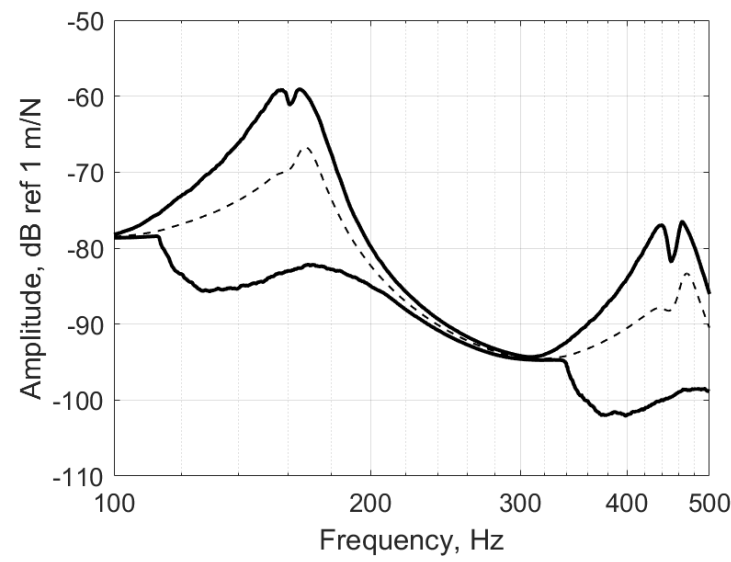

(a) $c_{l}=4 L_{t}$

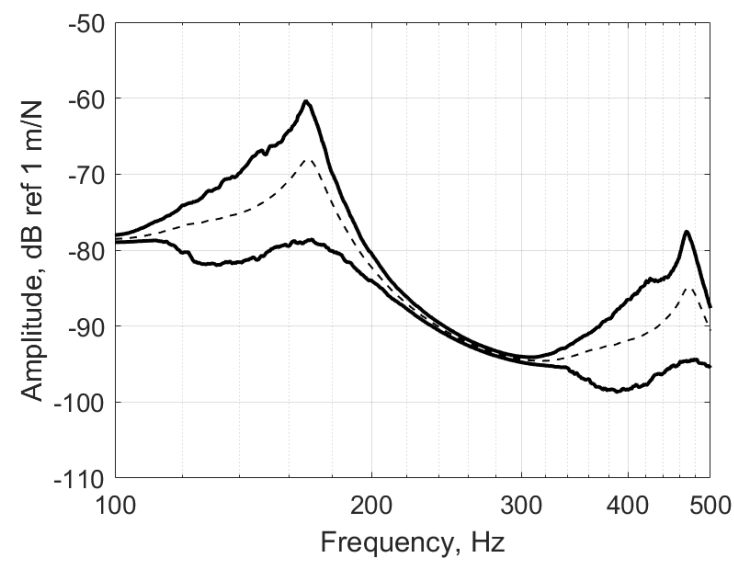

(c) $c_{l}=0.25 L_{t}$

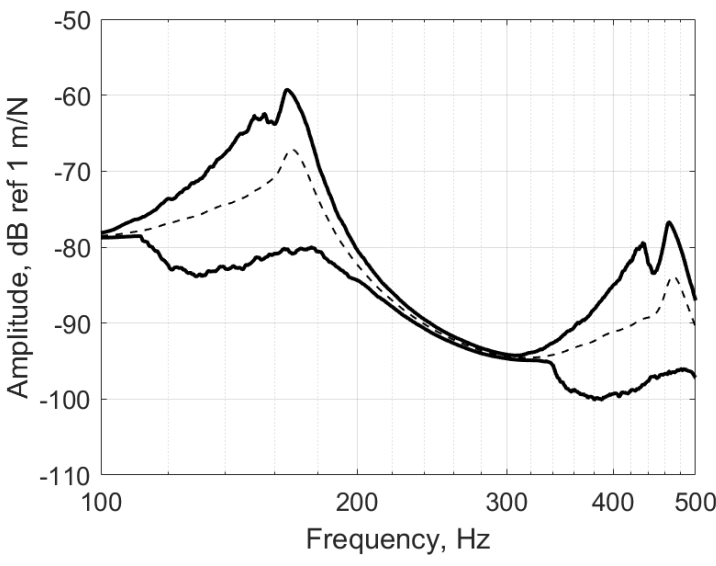

(b) $c_{l}=L_{t}$

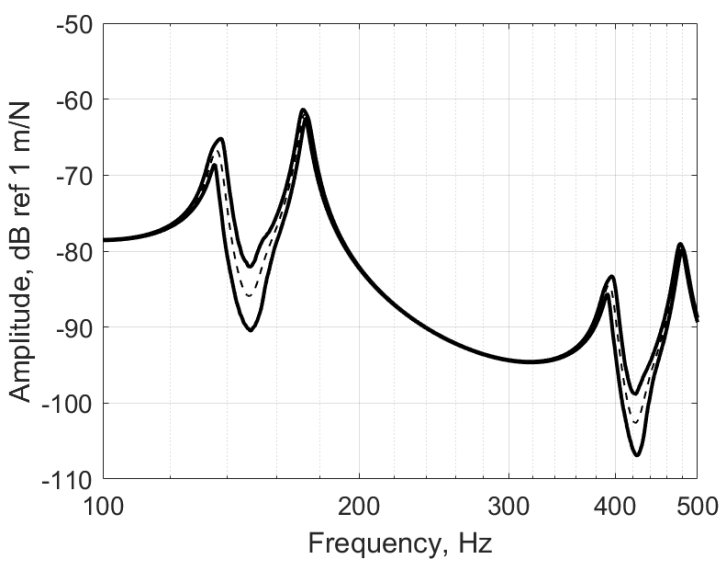

(d) $c_{l} \rightarrow 0$

Figure 10. Mean value (dashed line) and the $95^{t h}$ and $5^{t h}$ percentile of the amplitude of the transfer receptance of the metastructure.

structures with improved vibroacoustic performance, 2020. DOI:10.1016/j.ymssp.2020.106870.

[3] Liu Z, Zhang X, Mao Y et al. Locally Resonant Sonic Materials. Science 2000; 289(5485): 1734-1736. DOI: 10.1126/science.289.5485.1734. URL http://science. sciencemag. org/content/289/5485/1734.

[4] Brennan MJ. Some Recent Developments in Adaptive Tuned Vibration Absorbers/Neutralisers. Shock and Vibration 2006; 13(4-5): 531-543. DOI:10.1155/2006/ 563934.

[5] Sugino C, Xia Y, Leadenham S et al. A general theory for bandgap estimation in locally resonant metastructures. Journal of Sound and Vibration 2017; 406(Supplement C): 104-123. DOI:10.1016/j.jsv. 2017.06.004. URL http://ww.sciencedirect.com/ science/article/pii/S0022460X17304704. 1612. 03130.

[6] Tsakmakidis KL, Boardman AD and Hess O. 'Trapped rainbow' storage of light in metamaterials. Nature 2007; 450(7168): 397-401. DOI:10.1038/nature06285. URL https://www. nature.com/articles/nature06285.

[7] Zhu J, Chen Y, Zhu X et al. Acoustic rainbow trapping. Scientific Reports 2013; 3: 1728. DOI: 10.1038/srep01728. URL https://www.nature.com/ articles/srep01728.

[8] Tian Z and Yu L. Rainbow trapping of ultrasonic guided waves in chirped phononic crystal plates. Scientific Reports 2017; 7: 40004. DOI:10.1038/srep40004. URL https://www . nature.com/articles/srep40004.

[9] Sanchez-Dehesa J, Arreola-Lucas A, Baez G et al. Mechanical rainbow trapping and Bloch oscillations in chirped metallic beams. The Journal of the Acoustical Society of America 2017; 141(5): 3810. DOI:10.1121/1. 4988421. URL https://asa.scitation.org/doi/abs/ 10.1121/1.4988421.

[10] Meng H, Chronopoulos D, Fabro AT et al. Rainbow metamaterials for broadband multi-frequency vibration attenuation: Numerical analysis and experimental validation. Journal of Sound and Vibration 2020; 465: 115005. DOI:10.1016/j.jsv.2019.115005.

[11] Meng H, Chronopoulos D, Fabro AT et al. Optimal design of rainbow elastic metamaterials. International Journal of Mechanical Sciences 2019; : 105185DOI:10.1016/j.ijmecsci.2019.105185. URL http://www.sciencedirect.com/science/article/ pii/S0020740319322568.

[12] Thomes RL, Mosquera-Sánchez JA and De Marqui C. An investigation of optimal non-uniform locally 


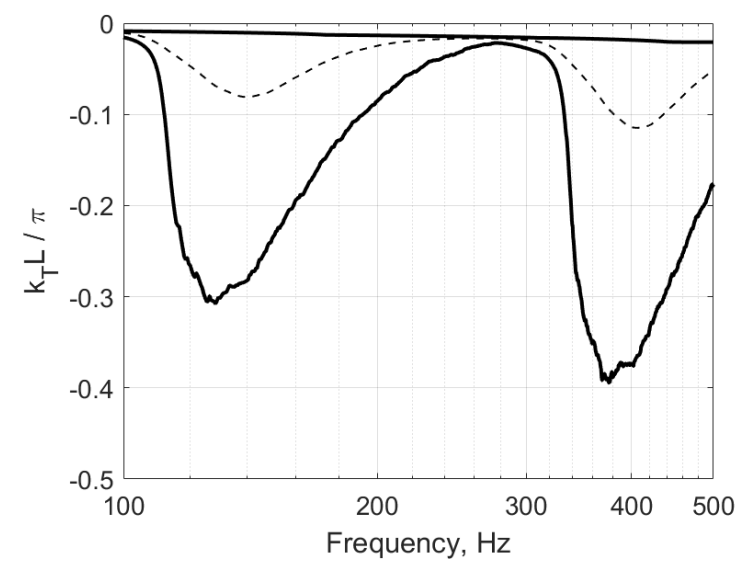

(a) $c_{l}=4 L_{t}$

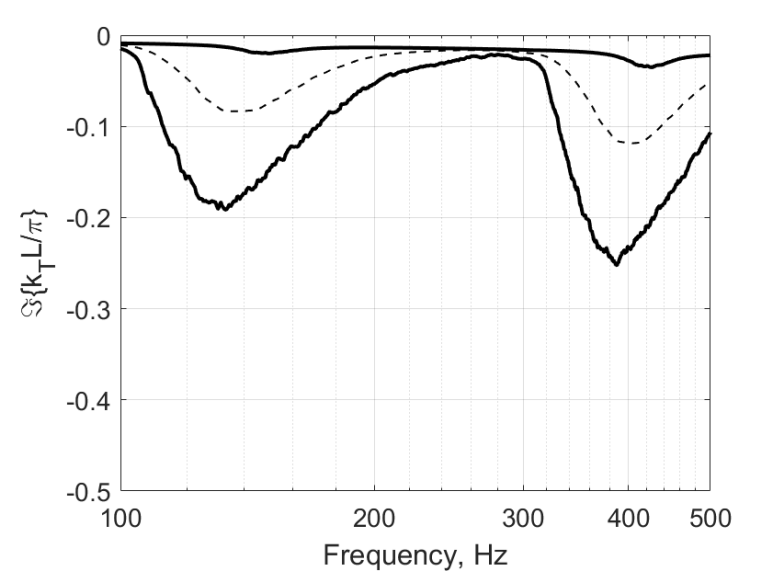

(c) $c_{l}=0.25 L_{t}$

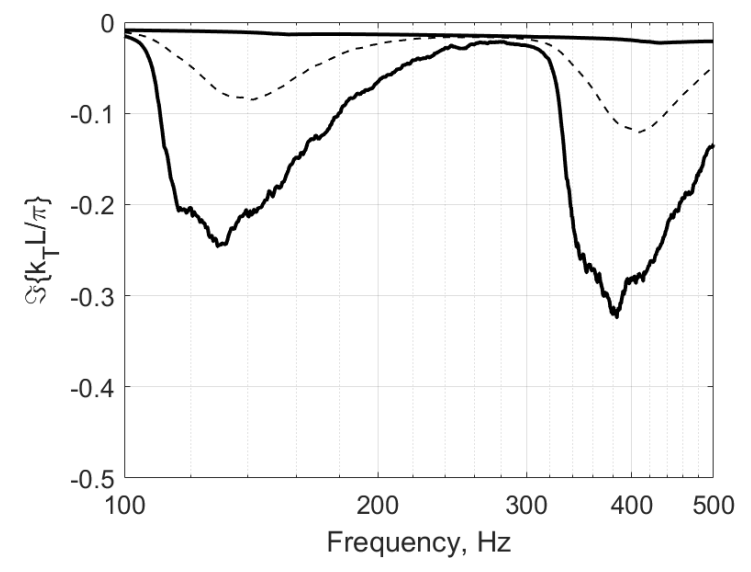

(b) $c_{l}=L_{t}$

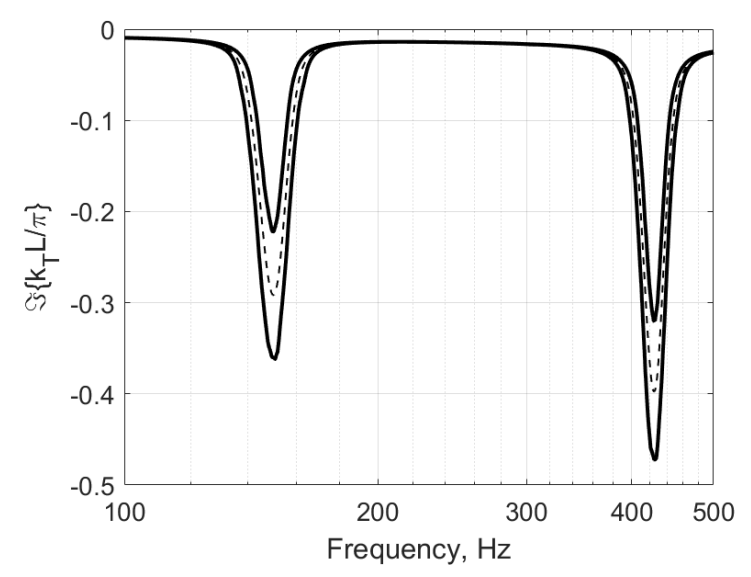

(d) $c_{l} \rightarrow 0$

Figure 11. Mean value (dashed line) and the $95^{t h}$ and $5^{t h}$ percentile of the imaginary part of the equivalent wavenumber $k_{T}$.

resonant piezoelectric metamaterials. In Han JH, Shahab S and Wang G (eds.) Active and Passive Smart Structures and Integrated Systems IX, volume 11376. SPIE. ISBN 9781510635296, p. 37. DOI:10.1117/12. 2558552.

[13] Chen AL and Wang YS. Study on band gaps of elastic waves propagating in one-dimensional disordered phononic crystals. Physica B: Condensed Matter 2007; 392(1-2): 369-378. DOI:10.1016/j.physb.2006.12.004.

[14] Timorian S, Petrone G, De Rosa S et al. Spectral analysis and structural response of periodic and quasiperiodic beams. Proceedings of the Institution of Mechanical Engineers, Part C: Journal of Mechanical Engineering Science 2019; 233(23-24): 7498-7512. DOI: 10.1177/0954406219888948. URL http://journals . sagepub.com/doi/10.1177/0954406219888948.

[15] Timorian S, Ouisse M, Bouhaddi N et al. Numerical investigations and experimental measurements on the structural dynamic behaviour of quasi-periodic metamaterials. Mechanical Systems and Signal Processing 2020; 136: 106516. DOI:10.1016/j.ymssp.2019.106516.

[16] Scott JFM. The statistics of waves propagating in a one-dimensional random medium. Proceedings of the Royal Society of London A Mathematical and Physical Sciences 1985; 398(1815): 341-363. DOI:10.1098/rspa.
1985.0038. URL https://royalsocietypublishing. org/doi/10.1098/rspa.1985.0038.

[17] Farzadian O and Niry MD. Delocalization of mechanical waves in the ladder chain of DNA with correlated disorder. Physica A: Statistical Mechanics and its Applications 2016; 450: 95-103. DOI:10.1016/j.physa. 2015.12.132.

[18] Costa AE and De Moura FA. Extended acoustic waves in diluted random systems. European Physical Journal B 2011; 80(1): 59-63. DOI:10.1140/epjb/ e2011-20016-3. URL https://link.springer.com/ article/10.1140/epjb/e2011-20016-3.

[19] Hodges CH and Woodhouse J. Confinement of vibration by one-dimensional disorder, I: Theory of ensemble averaging. Journal of Sound and Vibration 1989; 130(2): 237-251. DOI:10.1016/0022-460X(89)90552-X.

[20] Langley RS. Wave transmission through onedimensional near periodic structures: optimum and to random disorder. Journal of Sound and Vibration 1995; 188(5): 717-743. DOI:10.1006/jsvi.1995.0620.

[21] Langley RS, Bardell NS and Loasby PM. The optimal design of near-periodic structures to minimize vibration transmission and stress levels. Journal of Sound and Vibration 1997; 207(5): 627-646. DOI:10.1006/ jsvi.1997.1116. URL http://www.sciencedirect.com/ 
science/article/pii/S0022460X97911160.

[22] Baz A. Active control of periodic structures. Journal of Vibration and Acoustics, Transactions of the ASME 2001; 123(4): 472-479. DOI:10.1115/1.1399052.

[23] Ruzzene M and Baz A. Attenuation and localization of wave propagation in periodic rods using shape memory inserts. Smart Materials and Structures 2000; 9(6): 805. DOI:10.1088/0964-1726/9/6/310. URL http: //stacks. iop.org/0964-1726/9/i=6/a=310.

[24] Achaoui Y, Laude V, Benchabane S et al. Local resonances in phononic crystals and in random arrangements of pillars on a surface. Journal of Applied Physics 2013; 114(10): 104503. DOI:10.1063/ 1.4820928. URL http://aip.scitation.org/doi/10. 1063/1.4820928.

[25] Celli P, Yousefzadeh B, Daraio C et al. Bandgap widening by disorder in rainbow metamaterials. Applied Physics Letters 2019; 114(9): 091903. DOI:10.1063/ 1.5081916. URL http://aip.scitation.org/doi/10. 1063/1.5081916. 1703.08522.

[26] Meng H, Chronopoulos D and Fabro AT. Numerical simulation data for the dynamic properties of rainbow metamaterials. Data in Brief 2019; 104772DOI:10.1016/j.dib.2019.104772. URL http://www.sciencedirect.com/science/article/ $\mathrm{pii/S2352340919311278.}$

[27] Fabro AT, Meng $\mathrm{H}$ and Chronopoulos D. Uncertainties in the attenuation performance of a multifrequency metastructure from additive manufacturing. Mechanical Systems and Signal Processing 2020; 138: 106557. DOI:10.1016/j.ymssp. 2019.106557. URL http://www.sciencedirect.com/ science/article/pii/S0888327019307782.

[28] Luongo A. Mode localization by structural imperfections in one-dimensional continuous systems. Journal of Sound and Vibration 1992; 155(2): 249 271. DOI:10.1016/0022-460X(92)90510-5. URL http://www.sciencedirect.com/science/article/ pii/0022460X92905105.

[29] Mace BR and Manconi E. Wave motion and dispersion phenomena: Veering, locking and strong coupling effects. The Journal of the Acoustical Society of America 2012; 131(2): 1015-1028. DOI:10.1121/1. 3672647. URL https://asa.scitation.org/doi/10. 1121/1.3672647.

[30] Beli D, Fabro AT, Ruzzene $M$ et al. Wave attenuation and trapping in 3D printed cantilever-in-mass metamaterials with spatially correlated variability. Scientific Reports 2019; 9(1): 5617. DOI:10.1038/ s41598-019-41999-0. URL https://www. nature.com/ articles/s41598-019-41999-0.

[31] Fabro AT, Ferguson NS, Jain T et al. Wave propagation in one-dimensional waveguides with slowly varying random spatially correlated variability. Journal of Sound and Vibration 2015; 343: 20-48. DOI:10.1016/j. jsv.2015.01.013.

[32] Fabro AT, Ferguson NS and Mace BR. Wave propagation in slowly varying waveguides using a finite element approach. Journal of Sound and Vibration 2019; 442: 308-329. DOI:10.1016/j.jsv.
2018.11.004. URL http://www.sciencedirect.com/ science/article/pii/S0022460X18307569.

[33] Vanmarcke E. Random fields: Analysis and synthesis revised and expanded new edition. World Scientific Publishing Co., 2010. ISBN 9789814307598. DOI: $10.1142 / 5807$.

[34] Machado MR, Adhikari S, Dos Santos JMC et al. Estimation of beam material random field properties via sensitivity-based model updating using experimental frequency response functions. Mechanical Systems and Signal Processing 2018; 102(Supplement C): 180-197. DOI:10.1016/j.ymssp.2017.08. 039. URL http://www.sciencedirect.com/science/ article/pii/S0888327017304727.

[35] Mukhopadhyay T, Adhikari S and Batou A. Frequency domain homogenization for the viscoelastic properties of spatially correlated quasi-periodic lattices. International Journal of Mechanical Sciences 2019; 150: 784806. DOI:10.1016/j.ijmecsci.2017.09.004.

[36] Schuëller GI and Pradlwarter HJ. Uncertain linear systems in dynamics: Retrospective and recent developments by stochastic approaches. Engineering Structures 2009; 31(11): 2507-2517. DOI:10.1016/j.engstruct. 2009.07.005. URL http://www.sciencedirect.com/ science/article/pii/S0141029609002338.

[37] Ghanem R and Spanos PD. Stochastic Finite Elements: A Spectral Approach. Revised edition ed. Minneola, N.Y.: Dover Publications, 2012. ISBN 978-0-486-428185 .

[38] Phoon KK, Huang SP and Quek ST. Simulation of second-order processes using Karhunen-Loeve expansion. Computers \& Structures 2002; 80(12): 1049-1060. DOI:10.1016/S0045-7949(02)00064-0. URL http://www. sciencedirect.com/science/article/ pii/S0045794902000640.

[39] Phoon KK, Huang HW and Quek ST. Simulation of strongly non-Gaussian processes using Karhunen-Loeve expansion. Probabilistic Engineering Mechanics 2005; 20(2): 188-198. DOI:10.1016/j.probengmech.2005.05. 007. URL http://www.sciencedirect.com/science/ article/pii/S0266892005000123.

[40] Li LB, Phoon KK and Quek ST. Comparison between Karhunen-Loève expansion and translationbased simulation of non-Gaussian processes. Computers \& Structures 2007; 85(5-6): 264276. DOI:10.1016/j.compstruc.2006.10.010. URL http://www.sciencedirect.com/science/article/ pii/S0045794906003373.

[41] Huang SP, Quek ST and Phoon KK. Convergence study of the truncated Karhunen-Loeve expansion for simulation of stochastic processes. International Journal for Numerical Methods in Engineering 2001; 52(9): 1029-1043. DOI:10.1002/nme.255. URL http: //doi.wiley.com/10.1002/nme.255.

[42] Charmpis DC, Schuëller GI and Pellissetti MF. The need for linking micromechanics of materials with stochastic finite elements: A challenge for materials science. Computational Materials Science 2007; 41(1): 27-37. DOI:10.1016/j.commatsci.2007.02.014. 
[43] Der Kiureghian A and Ke JB. The stochastic finite element method in structural reliability. Probabilistic Engineering Mechanics 1988; 3(2): 83-91. DOI:10. 1016/0266-8920(88)90019-7.

[44] Stefanou G. The stochastic finite element method: Past, present and future, 2009. DOI:10.1016/j.cma.2008.11. 007.

[45] Stefanou G and Papadrakakis M. Assessment of spectral representation and Karhunen-Loève expansion methods for the simulation of Gaussian stochastic fields. Computer Methods in Applied Mechanics and Engineering 2007; 196(21-24): 2465-2477. DOI:10. 1016/j.cma.2007.01.009.

[46] de Cursi S and Sampaio R. Uncertainty Quantification and Stochastic Modeling with Matlab®. 1 edition ed. London: ISTE Press - Elsevier, 2015. ISBN 9780081004715. DOI:10.1016/C2014-0-04713-2.

[47] Rubinstein RY and Kroese DP. Simulation and the Monte Carlo Method. Wiley Series in Probability and Statistics, Hoboken, NJ, USA: John Wiley \& Sons, Inc., 2016. ISBN 9781118631980. DOI:10. 1002/9781118631980. URL http://doi.wiley.com/ $10.1002 / 9781118631980$. 